\title{
Misdiagnosis in occupational and environmental medicine: a scoping review
}

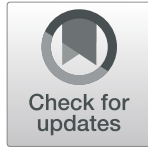

Jinyoung Moon ${ }^{1,2^{*}}$ (D) and HyeKyoung Yoo ${ }^{1}$

\begin{abstract}
Introduction: There has been no comprehensive review for misdiagnosis in Occupational and Environmental Medicine (OEM). The possible ramifications of an occupational disease (OD) or an environmental disease (ED) misdiagnosis are not just confined to the individual case but may extend to others exposed to the occupational or environmental hazard. Therefore, a comprehensive scoping review of published literature is imperative for understanding the nature of misdiagnoses in OEM.

Methods: A medical librarian searched MEDLINE (PubMed), EMBASE, and the Cochrane Library (on 06 November 2020). All collected OEM misdiagnoses were classified based on 2 conceptual frameworks, the typical framework, and the causation model. The distribution of misdiagnosis across each medical specialty, each diagnostic step of the typical framework and the causation model, and false-negative and false-positive were summarized.
\end{abstract}

Results: A total of 79 articles were included in the scoping review. For clinical specialty, pulmonology (30 articles) and dermatology or allergy (13 articles) was most frequent and second-most frequent, respectively. For each disease, occupational and environmental interstitial lung diseases, misdiagnosed as sarcoidosis (8 articles), and other lung diseases (8 articles) were most frequent. For the typical framework, the most vulnerable step was the first step, evidence of a disease (38 articles). For the causation model, the first step, knowledge base, was the most vulnerable step (42 articles). For reported articles, the frequency of false-negative (55 articles) outnumbered the frequency of false-positive (15 articles).

Discussion: In OEM, compared to general medicine, causal misdiagnosis associated with the probability of causation is also important. For making a diagnosis in OEM, a knowledge base about possible ODs and EDs is essential. Because of this reason, the education and training of treating physicians for common ODs and EDs are important. For ODs and EDs, various intentional behaviors of stakeholders should be considered. This scoping review might contribute to the improvement of understanding for misdiagnosis in OEM.

Keywords: Occupational and environmental medicine, Occupational disease, Environmental disease, Misdiagnosis, Scoping review

\footnotetext{
* Correspondence: pollux@snu.ac.kr

${ }^{1}$ Department of Environmental Health Science, Graduate School of Public Health, Seoul National University, Gwanak-ro 1, Gwanak-gu, Seoul 08826, South Korea

${ }^{2}$ Department of Occupational and Environmental Medicine, Seoul Saint

Mary's Hospital, Banpo-daero 222, Seocho-gu, Seoul 06591, South Korea
}

(c) The Author(s). 2021 Open Access This article is licensed under a Creative Commons Attribution 4.0 International License, which permits use, sharing, adaptation, distribution and reproduction in any medium or format, as long as you give appropriate credit to the original author(s) and the source, provide a link to the Creative Commons licence, and indicate if changes were made. The images or other third party material in this article are included in the article's Creative Commons licence, unless indicated otherwise in a credit line to the material. If material is not included in the article's Creative Commons licence and your intended use is not permitted by statutory regulation or exceeds the permitted use, you will need to obtain permission directly from the copyright holder. To view a copy of this licence, visit http://creativecommons.org/licenses/by/4.0/ The Creative Commons Public Domain Dedication waiver (http://creativecommons.org/publicdomain/zero/1.0/) applies to the data made available in this article, unless otherwise stated in a credit line to the data. 


\section{Introduction}

Misdiagnosis in medicine is encountered in everyday medical practice. Misdiagnosis is generally more common in clinical specialties (e.g., emergency medicine and internal medicine) than in perceptual specialties (e.g., radiology and pathology) [1]. In a study in Britain, about $6 \%$ of admitting diagnoses were incorrect in British hospitals [2]. The specialties requiring complex decisionmaking in settings of above-average uncertainty and stress (for example, emergency medicine) reported up to a $12 \%$ diagnostic error rate $[3,4]$. Based on lifelong studies on diagnostic decision-making, Arthur Elstein concluded that the rate of diagnostic error might be about $10-15 \%$ in overall medical practice [5].

Even if based on epidemiologic and toxicological principles, as a clinical specialty, occupational and environmental medicine (OEM) practice also encounters numerous instances of misdiagnosis. Compounding the opportunity for error, OEM practitioners have the added diagnostic step of detecting causality in addition to making the current medical diagnosis. Furthermore, the possible ramifications of an occupational disease (OD) or an environmental disease (ED) misdiagnosis are not just confined to the individual case but may extend to others exposed to the occupational or environmental hazard. Economic compensation and malpractice suits related to an OD or ED are other contributing factors to this complexity. For these reasons, the potential burden of misdiagnosis of an OD or ED could go beyond purely medical consequences, compared to typical misdiagnoses in general medicine. Because of these characteristics, various intentional behaviors of multiple stakeholders can possibly obscure the establishment of reliable causation between an occupational or environmental exposure and a related OD or ED [6-8].

To date, there has been no comprehensive review for misdiagnosis in OEM. However, with the potential wider implications of OD and ED considered a comprehensive review for this topic is imperative. In this scoping review, the authors tried to organize stepwise frameworks for the diagnosis of an OD and ED. Utilizing these frameworks, the authors analyzed and classified collected articles. In addition, the distributions of misdiagnoses in OEM through each specialty of medicine and by false-negative and false-positive misdiagnoses were also addressed. By examining the overall distribution patterns of misdiagnoses in OEM, the readers of this scoping review can understand patterns of misdiagnoses in OEM and devise possible preventive measures for reducing them.

\section{Methods}

\section{The definition of misdiagnosis in OEM}

When the concept of misdiagnosis in OEM is considered, the definition must include 2 categories. The first (i) is misdiagnosis from the general medical perspective, or medical misdiagnosis: the degree to which the diagnostic criteria for other medical diseases are fulfilled. The second (ii) is the misdiagnosis from the causal inference perspective, or causal misdiagnosis: the degree to which the occupational or environmental hazardous exposures contributed to the development of a disease. For this class of misdiagnosis, the probability of causation should exceed 50\% [9]. However, given the active criteria of published articles, the reporting of this second class of misdiagnosis in OEM is relatively scarce. Therefore, the main discussion of this class of misdiagnosis is reviewed in the discussion section.

\section{Research question of this scoping review}

The research question of this scoping review was to examine the distribution of misdiagnosis in OEM using published literature. This distribution included medical specialty, each step of diagnosis in OEM, and falsenegative/false-positive.

\section{Information sources and the selection of evidence sources}

A literature search was conducted by a medical librarian (information specialist, N.K. commented in the Acknowledgement section) in the library of one author's affiliation (Department of Occupational and Environmental Medicine, Seoul Saint Mary's Hospital). The medical librarian searched MEDLINE (PubMed), EMBASE, and the Cochrane Library (on 06 November 2020). Additionally, the authors searched the 3 databases on 08 January 2021 to complement the search results. Detailed search terminologies and search queries are provided from Supplementary A-1 to A-3.

The inclusion and exclusion criteria were as follows: (i) The article deals with a misdiagnosis case or an issue related to misdiagnosis. (ii) The misdiagnosis dealt with is an OD or an ED. (iii) The misdiagnosis should have a meaning in the present time, considering changes in diagnostic criteria and technologies with time. (iv) Both false-negative and false-positive misdiagnoses were included. (v) The publication year should be from 1990 to the present time. (vi) If an author reported the same set of misdiagnosis series in a number of articles, only the most recent one was included. (vii) Literature in all languages was included.

\section{Data items}

Study type, subject population, initial misdiagnosis, correct final diagnosis, whether the article deals with a false-negative or false-positive case, the specialty of the doctor who made the initial and final diagnosis were summarized. For the classification between falsenegative and false-positive, the OD or ED became the 
standpoint for classification. The content of each article was summarized in a Supplementary theoretical review and Supplementary materials. Possible corrective strategies were also summarized in the table.

\section{Classification of collected misdiagnoses}

All collected misdiagnoses were classified based on 2 conceptual frameworks for the classification of misdiagnoses in OEM, which were provided in subsection 2.1 and 2.2 of Supplementary theoretical review (Table 1).

\section{Data charting process}

The distribution of misdiagnosis across each medical specialty, each diagnostic step of the typical framework and the causation model, and false-negative and falsepositive were summarized in Table 3 , Table 4, and Table 5 , respectively.

\section{Results}

\section{Selection of evidence sources}

Detailed search processes are in Supplementary material A-4. By the medical librarian, a total of 1168 articles were searched. By the authors, a total of 799 articles were searched. After excluding duplication, the authors conducted a primary selection process using the title and abstract. After this process, only 262 articles, searched by the medical librarian, and 62 articles, searched by the authors, remained. A full-text review was conducted for these 262 and 62 articles. Finally, 76 articles remained. From the bibliographies of relevant articles, 3 articles were additionally searched. Finally, a total of 79 articles were included in this scoping review.

\section{The characteristics and summary of individual evidence source}

The characteristics of all included articles are summarized in Supplementary material D. The study period spread from 1967 to 2018 . For the specialty of the diagnosing doctor, the initial misdiagnosis category included OEM physicians only in 9 articles out of 79 articles, but the final correct diagnosis category included OEM physicians in 17 articles out of 79 articles. The summary of the final included articles is provided in Table 2. The most and second-most frequent type of study was casereport and case-series, respectively (27 and 25 articles). The third-most and fourth-most frequent type of study was narrative review and discussion paper, respectively (10 and 6 articles). The following study types were case-

Table 1 Typical framework and causation model for misdiagnoses of OD and ED

\begin{tabular}{|c|c|c|c|c|c|c|}
\hline \multicolumn{7}{|c|}{ Typical framework } \\
\hline & (i) Evidence of a disease & \multicolumn{2}{|c|}{ (ii) Evidence of hazardous exposures } & \multicolumn{3}{|c|}{ (iii) Evidence of causal relationship } \\
\hline Proper work & $\begin{array}{l}\text { Looking for any evidence of } \\
\text { a disease in a subpopulation } \\
\text { (workplace or community) }\end{array}$ & \multicolumn{2}{|c|}{$\begin{array}{l}\text { Extensively searching for the evidence of } \\
\text { possible hazardous exposures in the } \\
\text { workplace or community in which } \\
\text { patients developed }\end{array}$} & \multicolumn{3}{|c|}{$\begin{array}{l}\text { Based on so-far known medical and public health } \\
\text { knowledge, calculate the probability of causation }\end{array}$} \\
\hline Flaw & $\begin{array}{l}\text { Shortage in knowledge } \\
\text { about possible occupational } \\
\text { and environmental diseases } \\
\text { Misidentification of a disease }\end{array}$ & \multicolumn{2}{|c|}{$\begin{array}{l}\text { Shortage in knowledge about hazardous } \\
\text { occupational or environmental exposures } \\
\text { Shortage of information about exact } \\
\text { exposure status of a patient }\end{array}$} & \multicolumn{3}{|c|}{$\begin{array}{l}\text { Shortage in knowledge about the causal relationship } \\
\text { between a hazardous exposure and a disease outcome }\end{array}$} \\
\hline Consequences & $\begin{array}{l}\text { Missed diagnosis } \\
\text { OD or ED diagnosed as } \\
\text { another general medical } \\
\text { disease }\end{array}$ & \multicolumn{2}{|c|}{$\begin{array}{l}\text { OD or ED diagnosed as another general } \\
\text { medical disease } \\
\text { Sometimes the diagnosis itself could be } \\
\text { denied. }\end{array}$} & \multicolumn{3}{|c|}{$\begin{array}{l}\text { OD or ED diagnosed as another general medical } \\
\text { disease } \\
\text { The estimated probability of causation below 50\%: no } \\
\text { acknowledgment of an OD or ED }\end{array}$} \\
\hline \multicolumn{7}{|l|}{ Causation model } \\
\hline & (i) Knowledge base & (ii) Heuristics & $\begin{array}{l}\text { (iii) Complete work- } \\
\text { ups }\end{array}$ & $\begin{array}{l}\text { (iv) } \\
\text { Diagnosis }\end{array}$ & $\begin{array}{l}\text { (v) } \\
\text { Management }\end{array}$ & (vi) Feedback \\
\hline Proper work & $\begin{array}{l}\text { The first case in a similar } \\
\text { exposure group should be } \\
\text { examined meticulously with } \\
\text { the generation of the } \\
\text { knowledge base. }\end{array}$ & $\begin{array}{l}\text { The proper } \\
\text { context should } \\
\text { be generated } \\
\text { based on } \\
\text { recognized } \\
\text { findings. }\end{array}$ & $\begin{array}{l}\text { Complete work-ups } \\
\text { should be done for } \\
\text { the consideration of } \\
\text { sufficient differential } \\
\text { diagnoses. }\end{array}$ & $\begin{array}{l}\text { A valid } \\
\text { synthesis } \\
\text { should be } \\
\text { carried } \\
\text { out. }\end{array}$ & $\begin{array}{l}\text { Appropriate } \\
\text { treatments and } \\
\text { preventive } \\
\text { measures } \\
\text { should be } \\
\text { given. }\end{array}$ & $\begin{array}{l}\text { Appropriate feedback } \\
\text { should be given to } \\
\text { diagnosis and } \\
\text { management. }\end{array}$ \\
\hline Flaw & Shortage in knowledge Base & Faulty heuristics & Immature closure & $\begin{array}{l}\text { Faulty } \\
\text { synthesis }\end{array}$ & $\begin{array}{l}\text { Bad } \\
\text { management }\end{array}$ & $\begin{array}{l}\text { No policy or social } \\
\text { system for feedback }\end{array}$ \\
\hline Consequences & $\begin{array}{l}\text { The OD or ED diagnosis for a } \\
\text { similar exposure group } \\
\text { cannot be made. }\end{array}$ & $\begin{array}{l}\text { An initial } \\
\text { diagnosis will be } \\
\text { incorrect }\end{array}$ & $\begin{array}{l}\text { An important } \\
\text { differential diagnosis } \\
\text { cannot be included. }\end{array}$ & $\begin{array}{l}\text { The final } \\
\text { tentative } \\
\text { diagnosis } \\
\text { will be } \\
\text { incorrect. }\end{array}$ & $\begin{array}{l}\text { The treatment } \\
\text { and preventive } \\
\text { measures will } \\
\text { fail }\end{array}$ & $\begin{array}{l}\text { Proper feedbacks on } \\
\text { diagnostic processes } \\
\text { and a trial of other } \\
\text { management options } \\
\text { cannot be made. }\end{array}$ \\
\hline
\end{tabular}


Table 2 The summary of the final included articles

\begin{tabular}{|c|c|c|c|c|c|}
\hline Study type & $\begin{array}{l}\text { Number of } \\
\text { studies }\end{array}$ & $\begin{array}{l}\text { Clinical specialty } \\
\text { (final correct } \\
\text { diagnosis) }\end{array}$ & False $(+)$ or $(-)^{a}$ & Typical framework ${ }^{\mathbf{b}}$ & Causation model $^{\mathrm{c}}$ \\
\hline Case-report & 27 & $\begin{array}{l}\text { Pulmonology: } 11 \\
\text { Dermatology: } 1 \\
\text { Orthopedics or trauma: } \\
10 \\
\text { Other specialties: } 5\end{array}$ & $\begin{array}{l}\text { False (+): } 7 \\
\text { False }(-): 20\end{array}$ & $\begin{array}{l}\text { [1] Evidence of a disease: } 14 \\
\text { [2] Evidence of hazardous } \\
\text { exposures:12 } \\
\text { [3] Evidence of causal relationship: } \\
1\end{array}$ & $\begin{array}{l}\text { [1] Knowledge base: } \\
15 \\
\text { [2] Heuristics: } 6 \\
\text { [3] Complete work- } \\
\text { ups: } 4 \\
\text { [4] Diagnosis: } 1 \\
\text { [6] Feedback: } 1\end{array}$ \\
\hline Case-series & 25 & $\begin{array}{l}\text { Pulmonology: } 10 \\
\text { Dermatology: } 2 \\
\text { Other specialties: } 13\end{array}$ & $\begin{array}{l}\text { False }(+): 5 \\
\text { False }(-): 17 \\
\text { False }(+) \text { and }(-) \text { : } \\
3\end{array}$ & $\begin{array}{l}\text { [1] Evidence of a disease: } 12 \\
\text { [2] Evidence of hazardous } \\
\text { exposures: } 8 \\
\text { [3] Evidence of causal relationship: } \\
5\end{array}$ & $\begin{array}{l}\text { [1] Knowledge base: } \\
12 \\
\text { [2] Heuristics: } 4 \\
\text { [3] Complete work- } \\
\text { ups: } 6 \\
\text { [4] Diagnosis: } 3\end{array}$ \\
\hline Case-control study & 3 & Pulmonology: 3 & False $(-): 3$ & $\begin{array}{l}\text { [2] Evidence of hazardous } \\
\text { exposures: } 3\end{array}$ & [1] Knowledge base: 3 \\
\hline Cohort study & 3 & $\begin{array}{l}\text { Pulmonology: } 2 \\
\text { Other specialties: } 1\end{array}$ & $\begin{array}{l}\text { False }(+): 1 \\
\text { False }(-): 1 \\
\text { False }(+) \text { and }(-) \text { : } \\
1\end{array}$ & [1] Evidence of a disease: 3 & $\begin{array}{l}\text { [1] Knowledge base: } 1 \\
\text { [3] Complete work- } \\
\text { ups: } 1 \\
\text { [4] Diagnosis: } 1\end{array}$ \\
\hline $\begin{array}{l}\text { Surveillance data } \\
\text { analysis }\end{array}$ & 2 & $\begin{array}{l}\text { Pulmonology: } 1 \\
\text { Other specialties: } 1\end{array}$ & False (-): 2 & $\begin{array}{l}\text { [1] Evidence of a disease: } 1 \\
\text { [3] Evidence of causal relationship: } \\
1\end{array}$ & $\begin{array}{l}\text { [1] Knowledge base: } 1 \\
\text { [2] Heuristics: } 1\end{array}$ \\
\hline Survey & 2 & Other specialties: 2 & $\begin{array}{l}\text { False }(-): 1 \\
\text { False }(+) \text { and }(-) \text { : } \\
1\end{array}$ & $\begin{array}{l}\text { [1] Evidence of a disease: } 1 \\
\text { [3] Evidence of causal relationship: } \\
1\end{array}$ & $\begin{array}{l}\text { [2] Heuristics: } 1 \\
\text { [6] Feedback: } 1\end{array}$ \\
\hline Exposure assessment & 1 & Pulmonology: 1 & False $(-): 1$ & $\begin{array}{l}\text { [2] Evidence of hazardous } \\
\text { exposures: } 1\end{array}$ & [1] Knowledge base: 1 \\
\hline Discussion & 6 & $\begin{array}{l}\text { Pulmonology: } 3 \\
\text { Orthopedics or trauma: } \\
1 \\
\text { Other specialties: } 2\end{array}$ & $\begin{array}{l}\text { False }(+): 1 \\
\text { False }(-): 4 \\
\text { False }(+) \text { and }(-) \text { : } \\
1\end{array}$ & $\begin{array}{l}\text { [1] Evidence of a disease: } 2 \\
\text { [2] Evidence of hazardous } \\
\text { exposures:2 } \\
\text { [3] Evidence of causal relationship: } \\
2\end{array}$ & $\begin{array}{l}\text { [1] Knowledge base: } 1 \\
\text { [2] Heuristics: } 1 \\
\text { [3] Complete work- } \\
\text { ups: } 3 \\
\text { [4] Diagnosis: } 1\end{array}$ \\
\hline Narrative review & 10 & $\begin{array}{l}\text { Pulmonology: } 5 \\
\text { Orthopedics or trauma: } \\
1 \\
\text { Other specialties: } 4\end{array}$ & $\begin{array}{l}\text { False }(-): 7 \\
\text { False }(+) \text { and }(-) \text { : } \\
3\end{array}$ & $\begin{array}{l}\text { [1] Evidence of a disease: } 5 \\
\text { [2] Evidence of hazardous } \\
\text { exposures: } 5\end{array}$ & $\begin{array}{l}\text { [1] Knowledge base: } 8 \\
\text { [2] Heuristics: } 1 \\
\text { [4] Diagnosis: } 1\end{array}$ \\
\hline
\end{tabular}

${ }^{\mathrm{a}}$ False (+) or (-) for Occupational Disease or Environmental Disease Category.

${ }^{\mathrm{b}}$ Typical framework in the Methods section 2.5. Typical framework for the diagnosis of OD and ED.

${ }^{c}$ Causation model in the Methods section 2.6. Causation model for misdiagnosis of OD or ED.

control study, cohort study, surveillance data analysis, survey, and exposure assessment in order of frequency (3, 3, 2, 2, and 1 article, respectively). The summary and possible corrective strategies for each article are provided in Supplementary material B.

\section{Initial misdiagnosis, correct diagnosis, and the frequency} for each clinical specialty

When classified according to each clinical specialty (Table 3), misdiagnoses were reported most frequently in pulmonology (30 articles), followed by dermatology or allergy (13 articles). Reports in poisoning (10 articles) and orthopedics or trauma (10 articles) were also common.

For each disease, the most frequently reported type was chronic beryllium disease, silicosis, and other occupational interstitial lung diseases (ILD) misdiagnosed as sarcoidosis initially ( 8 articles) and occupational or environmental interstitial lung disease misdiagnosed as idiopathic pulmonary fibrosis or other lung diseases (8 articles). Poisoning was also commonly misdiagnosed as another disease (6 articles).

\section{Classification according to each step of the typical framework and causation model}

The searched articles were classified according to each step of the typical framework and causation model (Table 4). For the typical framework, the most vulnerable step was the first step, evidence of a disease (38 articles). The next vulnerable step was the second step, evidence of hazardous exposures (31 articles). The 
Table 3 Initial misdiagnosis, correct diagnosis, and the frequency for each clinical specialty

\begin{tabular}{|c|c|c|c|}
\hline $\begin{array}{l}\text { Clinical specialty } \\
\text { (frequency) }\end{array}$ & Initial misdiagnosis & Correct diagnosis & Frequency \\
\hline \multirow{10}{*}{$\begin{array}{l}\text { Dermatology or } \\
\text { Allergy (13 articles) }\end{array}$} & Non-allergic irritant contact dermatitis & Allergic contact dermatitis (Occupational epoxy) & 2 \\
\hline & Allergic contact dermatitis & Non-allergic contact dermatitis & 3 \\
\hline & Laryngopharyngeal reflux & Allergic laryngitis (Occupational origin) & 1 \\
\hline & Asthma & Irritant vocal cord dysfunction & 2 \\
\hline & Reactive airway dysfunction syndrome & & \\
\hline & Occupational asthma & No asthmatic reaction & 1 \\
\hline & Asthma or exercise-induced bronchoconstriction & No lung function abnormality & 1 \\
\hline & Bronchial asthma (occupational origin) & Carcinoid syndrome & 1 \\
\hline & Other respiratory diseases & Occupational asthma & 2 \\
\hline & No asthmatic reaction & & \\
\hline \multirow{15}{*}{$\begin{array}{l}\text { Pulmonology (30 } \\
\text { articles) }\end{array}$} & Sarcoidosis & Chronic beryllium disease & 8 \\
\hline & & Silicosis & \\
\hline & & Occupational interstitial lung disease & \\
\hline & Asthma or asthmatic bronchitis & Hypersensitivity pneumonitis & 2 \\
\hline & Idiopathic interstitial lung disease & & \\
\hline & Pneumoconiosis or other lung diseases & Pneumoconiosis or other lung diseases & 2 \\
\hline & $\begin{array}{l}\text { Idiopathic pulmonary fibrosis or other lung } \\
\text { diseases (including Tuberculosis) }\end{array}$ & $\begin{array}{l}\text { Occupational or environmental interstitial lung disease } \\
\text { (pneumoconiosis, silicosis, silicotuberculosis, or pigeon fancier's } \\
\text { lung) }\end{array}$ & 8 \\
\hline & Other lung diseases & Chronic terminal airways and parenchymal lung disease, & 2 \\
\hline & Other lung diseases & & \\
\hline & Typical lung cancer & Occupational lung cancer & 1 \\
\hline & Asbestos-related malignant cancer & Bilateral parietal pleural plaque & 3 \\
\hline & Benign asbestos pleurisy & Malignant mesothelioma & 2 \\
\hline & Thoracic aortic rupture or dissection & & \\
\hline & Drug-resistant pneumonia & Allergic bronchopulmonary aspergillosis & 1 \\
\hline & Chronic beryllium disease & Mycobacterium infection (Avium intracellulare) & 1 \\
\hline \multirow[t]{4}{*}{$\begin{array}{l}\text { Poisoning }(10 \\
\text { articles) }\end{array}$} & Other diseases & $\begin{array}{l}\text { Poisoning (Lead, Methyl iodide and manganese, N-hexane, and } \\
\text { Mercury) }\end{array}$ & 6 \\
\hline & $\begin{array}{l}\text { Neurotoxicant exposure or primary psychiatric } \\
\text { illness and multiple chemical sensitivity }\end{array}$ & $\begin{array}{l}\text { Neurotoxicant exposure or primary psychiatric illness and } \\
\text { multiple chemical sensitivity }\end{array}$ & 1 \\
\hline & Neurotoxic disease & $\begin{array}{l}\text { A naturally occurring nervous system disease, psychogenic } \\
\text { illness (including cognitive malingering) }\end{array}$ & 2 \\
\hline & Viral flu & Polymer fume fever & 1 \\
\hline \multirow{10}{*}{$\begin{array}{l}\text { Orthopedics or } \\
\text { trauma (10 articles) }\end{array}$} & Occupational overuse syndrome & Guyon's canal syndrome & 1 \\
\hline & Other bone abnormalities & Condensing osteitis of the clavicle & 1 \\
\hline & Motor dysfunction disease & Musician's focal dystonia & 1 \\
\hline & Ulnar styloid fracture & Calcific tendinitis of the flexor carpi ulnaris & 1 \\
\hline & $\begin{array}{l}\text { Occupational injury and illness or Other diseases } \\
\text { of non-occupational origin }\end{array}$ & $\begin{array}{l}\text { Occupational injury and illness or Other diseases of non- } \\
\text { occupational origin }\end{array}$ & 1 \\
\hline & Carpal tunnel syndrome & Nonspecific activity-related arm pain & 1 \\
\hline & Hand-arm vibration syndrome & Cold hemagglutinin disease & 1 \\
\hline & Fractured orbital bone & A plastic foreign body (Occupational craniofacial injury) & 2 \\
\hline & Retrobulbar hemorrhage/edema & & \\
\hline & A spiral fracture of the right tibia & Unrecognized foreign body & 1 \\
\hline Infection (5 articles) & Toxoplasmosis & Whipple's disease & 1 \\
\hline
\end{tabular}


Table 3 Initial misdiagnosis, correct diagnosis, and the frequency for each clinical specialty (Continued)

\begin{tabular}{|c|c|c|c|}
\hline $\begin{array}{l}\text { Clinical specialty } \\
\text { (frequency) }\end{array}$ & Initial misdiagnosis & Correct diagnosis & Frequency \\
\hline & An insect bite or Child abuse & Phytophotodermatitis & 1 \\
\hline & Other febrile diseases. & Tsutsugamushi disease (occupational origin) & 1 \\
\hline & Varicella-zoster virus infection & No infection & 1 \\
\hline & Virus infection & Leptospirosis & 1 \\
\hline \multirow[t]{5}{*}{ Others (5 articles) } & Acute kidney injury & Normal kidney function & 1 \\
\hline & $\begin{array}{l}\text { Occupational noise-induced hearing loss or } \\
\text { other hearing loss disease }\end{array}$ & $\begin{array}{l}\text { Occupational noise-induced hearing loss or other hearing loss } \\
\text { disease }\end{array}$ & 1 \\
\hline & Other psychiatric or stress disorder & Workplace adjustment disorder & 1 \\
\hline & Arterial gas embolism & Facial nerve baroparesis & 1 \\
\hline & Pelvic infection & Corpus cavernosum thrombosis & 1 \\
\hline \multirow[t]{3}{*}{ General (6 articles) } & Other diseases & Occupational diseases, general & 3 \\
\hline & $\begin{array}{l}\text { Environmental illnesses (including Minamata } \\
\text { disease) or other diseases }\end{array}$ & Environmental illnesses or other diseases & 2 \\
\hline & Asthma or allergic condition & Multiple chemical sensitivity & 1 \\
\hline
\end{tabular}

following vulnerable step was the third step, evidence of causal relationship (10 articles).

For the causation model, the first step, knowledge base, was the most vulnerable step (42 articles). The next was the complete work-ups step (14 articles) and the heuristics step (14 articles). Diagnosis (7 articles) and feedback (2 articles) steps also reported misdiagnosis. The articles classified in each step are listed in Supplementary material C-1 and C-2.

\section{Classification according to false-negative and false- positive}

The searched articles were classified according to falsenegative and false-positive (Table 5). For reported articles, the frequency of false-negative (55 articles) outnumbered the frequency of false-positive (15 articles). Some articles reported misdiagnoses that could be falsenegative or false-positive according to circumstances (9 articles). For example, chronic beryllium disease can be diagnosed as sarcoidosis, which is false-negative. In contrast, the pulmonary infection of $M$. avium intracellulare can be diagnosed as chronic beryllium disease, which is false-positive. As another example, occupational noise- induced hearing loss can be diagnosed as other sensorineural hearing losses, which is false-negative. In contrast, other sensorineural hearing losses can be diagnosed as occupational noise-induced hearing loss, which is falsepositive.

\section{Discussion}

In this scoping review, OEM misdiagnoses reported in published literature were summarized (a total of 79 articles). The major study type was case report and case series (27 and 25 articles, respectively). The initial diagnosis team included OEM physicians only in 9 articles, but the final diagnosis team included OEM physicians in 17 articles. For clinical specialty, pulmonology (30 articles) and dermatology or allergy (13 articles) specialty were most frequent. For each disease, occupational and environmental interstitial lung diseases (ILD), misdiagnosed as sarcoidosis ( 8 articles), and other lung diseases ( 8 articles) were most frequent. For the typical framework, the most vulnerable step was the first step, evidence of a disease (38 articles). For the causation model, the first step, knowledge base, was the most vulnerable step (42 articles). For reported articles, the frequency of

Table 4 Classification according to each step of two diagnostic models

\begin{tabular}{|c|c|c|c|c|c|c|}
\hline \multicolumn{7}{|l|}{ Typical framework } \\
\hline \multirow{2}{*}{$\begin{array}{l}\text { Each step of the typical framework } \\
\text { Frequency }\end{array}$} & \multicolumn{2}{|c|}{ (i) Evidence of a disease } & \multicolumn{2}{|c|}{$\begin{array}{l}\text { (ii) Evidence of hazardous } \\
\text { exposures }\end{array}$} & \multicolumn{2}{|c|}{$\begin{array}{l}\text { (iii) Evidence of causal } \\
\text { relationship }\end{array}$} \\
\hline & 38 & & 31 & & 10 & \\
\hline \multicolumn{7}{|l|}{ Causation model } \\
\hline Each step of the causation model & (i) Knowledge base & (ii) Heuristics & (iii) Complete work-ups & (iv) Diagnosis & (v) Management & (vi) Feedback \\
\hline Frequency & 42 & 14 & 14 & 7 & 0 & 2 \\
\hline
\end{tabular}


Table 5 Misdiagnosis according to false-negative and false-positive

\begin{tabular}{|c|c|c|c|}
\hline $\begin{array}{l}\text { Clinical specialty } \\
\text { (frequency) }\end{array}$ & False-negative (55 articles) & $\begin{array}{l}\text { Both false-negative and -positive ( } 9 \\
\text { articles) }\end{array}$ & False-positive (15 articles) \\
\hline Pulmonology & $\begin{array}{l}\text { Occupational asthma [10, 11] } \\
\text { Chronic beryllium disease [12-16] } \\
\text { Hypersensitivity pneumonitis [17-19] } \\
\text { Pigeon fancier's lung [20] } \\
\text { Obliterative bronchiolitis [21] } \\
\text { Malignant mesothelioma [22] } \\
\text { Occupational lung cancer [23] } \\
\text { Silicosis [24-27] } \\
\text { Interstitial lung disease [28-32] } \\
\text { Mesothelioma [33, 34] } \\
\text { Allergic bronchopulmonary aspergillosis } \\
\text { [35] }\end{array}$ & $\begin{array}{l}\text { Pneumoconiosis [65] } \\
\text { Interstitial lung disease }[66,67] \\
\text { Asbestosis [68] }\end{array}$ & $\begin{array}{l}\text { Occupational asthma }[74,75] \\
\text { Bronchial asthma [76] } \\
\text { Asbestos-related cancer [77] } \\
\text { Chronic beryllium disease }[78]\end{array}$ \\
\hline Dermatology & $\begin{array}{l}\text { Allergic contact dermatitis [36, 37] } \\
\text { Phytophotodermatitis [38] } \\
\text { Allergic laryngitis [39] }\end{array}$ & & $\begin{array}{l}\text { Latex allergy reaction [79] } \\
\text { Allergic contact dermatitis }[80,81]\end{array}$ \\
\hline Orthopedics or trauma & $\begin{array}{l}\text { Musician's focal dystonia [40] } \\
\text { Calcific tendinitis of the flexor carpi } \\
\text { ulnaris [41] } \\
\text { A foreign body [42-44] } \\
\text { Condensing osteitis in the clavicle [45] }\end{array}$ & Workplace injury and illness [69] & $\begin{array}{l}\text { Hand-arm vibration syndrome [82] } \\
\text { Occupational overuse syndrome [83] } \\
\text { Carpal tunnel syndrome [84] }\end{array}$ \\
\hline Other clinical specialties & $\begin{array}{l}\text { Tsutsugamushi disease [46] } \\
\text { Methyl iodide and Manganese } \\
\text { poisoning [47] } \\
\text { N-hexane poisoning [48] } \\
\text { Lead poisoning [49, 50] } \\
\text { Mercury poisoning [51,52] } \\
\text { Polymer fume fever [53] } \\
\text { Irritant vocal cord dysfunction [54, 55] } \\
\text { Multiple chemical sensitivity [56] } \\
\text { Adjustment disorder [57] } \\
\text { Whipple's disease [58] } \\
\text { Occupational disease, general [59-61] } \\
\text { Facial nerve baroparesis [62] } \\
\text { Leptospirosis [63] } \\
\text { Corpus carvernosum thrombosis [64] }\end{array}$ & $\begin{array}{l}\text { Noise-induced hearing loss [70] } \\
\text { Minamata disease [71] } \\
\text { Multiple chemical sensitivity [72] } \\
\text { Environmental illness [73] }\end{array}$ & $\begin{array}{l}\text { Acute kidney injury [85] } \\
\text { Varicella-zoster virus infection [86] } \\
\text { Neurotoxic disease [87] } \\
\text { Cognitive malingering in toxic } \\
\text { exposure [88] }\end{array}$ \\
\hline
\end{tabular}

The false-negative or false-positive was determined using an occupational or environmental disease as the standard.

false-negative (55 articles) outnumbered the frequency of false-positive (15 articles).

\section{'Medical misdiagnosis' versus 'causal misdiagnosis': the probability of causation}

As stated in subsection 2.1, misdiagnoses in OEM are classified into 2 classes: 'medical misdiagnosis' and 'causal misdiagnosis.' The published articles usually focused on the first 'medical misdiagnosis' cases, and 'causal misdiagnosis' cases were scarcely reported. The reason for this might be the difficulty in calculating a correct probability of causation [9]. The 'medical misdiagnosis' is rather clearly defined and can be identified easily. However, the 'causal misdiagnosis' is the main area in which various disputes about compensation occur [89]. Case by case and physician by physician, the calculated probability of causation could be different, and this differently calculated probability of causation causes a different decision whether or not that this disease is of an occupational or environmental origin.
Detailed discussion is provided in subsection 3.1. of Supplementary theoretical review.

\section{Misdiagnosis in general medicine versus misdiagnosis in OEM}

Compared to our analysis showing the most frequent step for misdiagnoses in OEM as the first 'knowledge base' step, misdiagnoses in general medicine were most frequent in the 'synthesis of collected information' step [90], which corresponds to the second, third, and fourth steps in our causation model. This difference is because OEM usually uses the type 2 systematic and analytic approach to make a diagnosis, while general medicine also uses the type 1 heuristic and intuitive approach more commonly than OEM [91]. This diagnostic feature of OEM makes the education and training of treating physicians (including general physicians) for the clinical manifestations and diagnostic clues of various occupational and environmental exposures essential [92]. Because of this diagnostic feature of OEM, the initial 
misdiagnosis category included OEM physicians as the diagnosing physician only in 9 articles out of 79 articles, but the final correct diagnosis category included OEM physicians as the diagnosing physician in 17 articles out of 79 articles in this study. Given that OEM physicians usually have more knowledge about the clinical manifestations and diagnostic clues of various ODs and EDs, this result can be understood.

\section{Intentional behaviors of stakeholders}

An important feature of ODs and EDs is that there are various intentional behaviors of stakeholders [93]. Because practical benefits like worker's compensation or accident and sickness benefits exist in most countries, cognitive malingering could be prevalent in a specific workplace or country. This also causes false-positive cases. On the other hand, for employers, concealing industrial accidents or occupational diseases is favorable for their profits. Therefore, they usually try to elucidate no relationship between a specific working environment and an OD. This is the same for most EDs [94]. For these ED cases, the citizen takes the role of workers, and the company or the government who is responsible for having made an environmental hazard acts in the role of employer.

In addition, some environmental illnesses do not have definitive diagnostic criteria, and this causes both falsenegative and -positive misdiagnoses [71-73]. As the solution for Minamata disease [71], a quantitative score can be instituted for diagnosis. Discriminant values in principal component analysis or classic machine learning methods are good examples of calculating this score.

\section{Risk of bias: case report and case series studies}

This scoping review included 25 case report studies and 25 case series studies, among 79 total included studies. With case reports and case series that have no comparison group, one cannot conclude the magnitude or the frequency of a particular type of misdiagnosis [95]. However, these study types could provide an overall picture of misdiagnosis profile in OEM. This scoping review could be a foundation for future quantitative studies about particular types of misdiagnoses in OEM.

\section{Other limitations of this study}

Several limitations exist in this scoping review. First, this study only included published misdiagnosis cases. Therefore, there will be some degree of publication bias in reported misdiagnosis cases. In particular, misdiagnoses with significant consequences for the treating physician might not be reported in the literature. In addition, considering the aforementioned confusion between the probability of causation and relative risk, acknowledged OD or ED might represent only a small percentage of overall true OD or EDs. Of the 3 categories of misdiagnosis aforementioned, only the first and second categories would have been reported in the literature. The third 'missed diagnosis' category should receive greater scrutiny in future research.

Second, there would be numerous misdiagnosis cases that were not revealed because of dynamics in workplaces or intentional behaviors of employers or employees. Sometimes, the government of a country is unfavorable to OD or EDs for economic growth (particularly developing countries). In this culture, the diagnosis of an OD or ED cannot be properly made.

\section{Conclusion}

In this scoping review, we surveyed the distribution of misdiagnosis articles through each medical specialty, false-negative or -positive, and each diagnostic step of OEM. In the discussion, several related concepts are discussed. In OEM, in contrast to general medicine, causal misdiagnosis associated with the probability of causation is also important. For making a diagnosis in OEM, a knowledge base about possible ODs and EDs is essential. Because of this reason, the education and training of treating physicians for common ODs and EDs are important. For ODs and EDs, various intentional behaviors of stakeholders should be considered. This scoping review might contribute to the improvement of understanding misdiagnosis in OEM.

\section{Supplementary Information}

The online version contains Supplementary theoretical review and Supplementary materials available at https://doi.org/10.1186/s12995-02100325-z.

Additional file 1. Supplementary theoretical review

Additional file 2. Supplementary materials

\begin{abstract}
Acknowledgments
The authors appreciate the work of Najin Kim in the Medical Library of the Catholic University of Korea, Seoul, Republic of Korea (ORCID: https://orcid. org/0000-0001-7280-9579), for building the search queries, searching the literature, and checking out the finally selected articles.

The authors appreciate the reviewers of this article. Their comments were of great help in improving the composition and quality of this article.
\end{abstract}

\section{Disclosure}

The authors have no potential conflicts of interest to disclose.

\section{Authors' contributions}

Jinyoung Moon (the corresponding and first author): Conceptualization, Methodology, Software, Validation, Formal analysis, Writing - Original Draft, Writing -Review \& Editing, Visualization, Supervision, Project administration. HyeKyoung Yoo (the second author): Investigation, Resources, Data Curation. The authors read and approved the final manuscript. public, commercial, or not-for-profit sectors. 


\section{Availability of data and materials}

This article is a scoping review, and all data and materials can be acquired from the published articles searched in 3 databases (PUBMED, EMBASE, and the Cochrane Library).

\section{Declarations}

Ethics approval and consent to participate

Not applicable.

\section{Consent for publication}

Not applicable.

\section{Competing interests}

The authors declare that they have no known competing financial interests or personal relationships that could have appeared to influence the work reported in this paper.

\section{Received: 18 April 2021 Accepted: 11 August 2021}

Published online: 24 August 2021

\section{References}

1. Berner ES, Graber ML. Overconfidence as a cause of diagnostic error in medicine. Am J Med. 2008;121(5):S2-S23. https://doi.org/10.1016/j.amjmed.2 008.01.001.

2. Neale G, Woloshynowych M, Vincent C. Exploring the causes of adverse events in NHS hospital practice. J R Soc Med. 2001;94(7):322-30. https://doi. org/10.1177/014107680109400702.

3. Chellis M, Olson JE, Augustine J, Hamilton GC. Evaluation of missed diagnoses for patients admitted from the emergency department. Acad Emerg Med. 2001;8(2):125-30. https://doi.org/10.1111/j.1553-2712.2001.tb012 76.x.

4. O'Connor P, Dowey K, Bell P, Irwin S, Dearden C. Unnecessary delays in accident and emergency departments: do medical and surgical senior house officers need to vet admissions? Emerg Med J. 1995;12(4):251-4. https://doi.org/10.1136/emj.12.4.251.

5. Higgs J, Jones MA, Loftus S, Christensen N. Clinical reasoning in the health professions E-book: Elsevier health sciences; 2008.

6. Dewitte J, Chan-Yeung M, Malo J. Medicolegal and compensation aspects of occupational asthma. Eur Respir J. 1994;7(5):969-80.

7. Postol LP. Suing the doctor: lawsuits by injured workers against the occupational physician. J Occup Med. 1989;31(11):891-6. https://doi.org/10.1 097/00043764-198911000-00009.

8. Johnstone RT. Occupational diseases. Diagnosis, Medicolegal Aspects and Treatment. Occupational Diseases Diagnosis, Medicolegal Aspects and Treatment; 1941.

9. Greenland S. Relation of probability of causation to relative risk and doubling dose: a methodologic error that has become a social problem. Am J Public Health. 1999;89(8):1166-9. https://doi.org/10.2105/AJPH.89.8.11 66.

10. Sastre J, Fernandez-Nieto M, Novalbos A, De Las HM, Cuesta J, Quirce S. Need for monitoring nonspecific bronchial hyperresponsiveness before and after isocyanate inhalation challenge. Chest. 2003;123(4):1276-9. https://doi. org/10.1378/chest.123.4.1276

11. Constantin B, Postolache P, Croitoru A, Nemes R. Occupational bronchial asthma-clinical and epidemiological aspects. J Environ Prot Ecol. 2015;16(2): 517-20

12. Fireman E, Haimsky E, Noiderfer M, Priel I, Lerman Y. Misdiagnosis of sarcoidosis in patients with chronic beryllium disease. Sarcoidosis Vasc Diffuse Lung Dis. 2003;20(2):144-8.

13. Laczniak AN, Gross NA, Fuortes LJ, Field RW. Unsuspected exposure to beryllium: potential implications for sarcoidosis diagnoses. Sarcoidosis Vasc Diffuse Lung Dis. 2014;31(2):163-9.

14. Müller-Quernheim J, Gaede K, Fireman E, Zissel G. Diagnoses of chronic beryllium disease within cohorts of sarcoidosis patients. Eur Respir J. 2006; 27(6):1190-5. https://doi.org/10.1183/09031936.06.00112205.

15. Kotloff RM, Richman PS, Greenacre JK, Rossman MD. Chronic beryllium disease in a dental laboratory technician. Am Rev Respir Dis. 1993;147(1): 205-7. https://doi.org/10.1164/ajrccm/147.1.205.

16. Baur X, Muller-Quernheim J. Case Report with Differential Diagnostic Aspects of Sarcoidosis. Pneumologie (Stuttgart, Germany). 2016;70(3):201-4.
17. Jacobs RL, Andrews CP, Coalson JJ. Hypersensitivity pneumonitis: beyond classic occupational disease-changing concepts of diagnosis and management. Ann Allergy Asthma Immunol. 2005;95(2):115-28. https://doi. org/10.1016/S1081-1206(10)61200-8.

18. Riario Sforza GG, Marinou A. Hypersensitivity pneumonitis: a complex lung disease. Clin Mol Allergy. 2017:15(1):6.

19. Ohtani Y, Ochi J, Mitaka K, Takemura T, Jinta T, Kuramochi J, et al. Chronic summer-type hypersensitivity pneumonitis initially misdiagnosed as idiopathic interstitial pneumonia. Intern Med. 2008;47(9):857-62. https://doi. org/10.2169/internalmedicine.47.0656

20. Rosal-Sanchez M, Alvarez J, Torres MJ, Mayorga C, Perez J, Blanca M. Pigeon Fancier's lung after low exposure. Allergy. 2002;57(7):649. https://doi.org/10.1 034/j.1398-9995.2002.t01-1-23736.x.

21. Centers for Disease C, Prevention. Obliterative bronchiolitis in workers in a coffee-processing facility - Texas, 2008-2012. MMWR Morb Mortal Wkly Rep. 2013;62(16):305-7

22. Mouawad NJ, Daniel VC, Starr JE. Advanced malignant mesothelioma mimicking acute contained thoracic aortic rupture. Interact Cardiovasc Thorac Surg. 2014;18(2):242-4. https://doi.org/10.1093/icvts/ivt465.

23. Kuratsune M, Tokudome S, Shirakusa T, Yoshida M, Tokumitsu Y, Hayano T, et al. Occupational lung cancer among copper smelters. Int J Cancer. 1974; 13(4):552-8. https://doi.org/10.1002/ijc.2910130415.

24. Kerget B, Araz O, Yilmazel Ucar E, Karaman A, Calik M, Alper F, et al. Female workers' silicosis diagnosis delayed due to gender bias. Occup Med (Oxford, England). 2019;69(3):219-22.

25. Guarnieri G, Bizzotto R, Gottardo O, Velo E, Cassaro M, Vio S, et al. Multiorgan accelerated silicosis misdiagnosed as sarcoidosis in two workers exposed to quartz conglomerate dust. Occup Environ Med. 2019;76(3):17880. https://doi.org/10.1136/oemed-2018-105462

26. Kerget B, Araz O, Ucar EY, Karaman A, Alper F, Akgun M. Delayed Diagnosis of Silicosis to Due Denim Sandblasting in a Female Worker. B49. OCCUPATIONAL EXPOSURES. American Thoracic Society International Conference Abstracts: American Thoracic Society; 2018. A3528-A3528.

27. Lü X, Wang H. Misdiagnosis of pneumoconiosis or silicotuberculosis in China: a pooled analysis of 1178 cases. Zhonghua lao dong wei sheng zhi ye bing za zhi= Zhonghua laodong weisheng zhiyebing zazhi= Chinese journal of industrial hygiene and occupational diseases. 2013;31(8):564-7.

28. Sauler M, Gulati M. Newly recognized occupational and environmental causes of chronic terminal airways and parenchymal lung disease. Clin Chest Med. 2012;33(4):667-80. https://doi.org/10.1016/j.ccm.2012.09.002.

29. Garland FC, Gorham ED, Kaiser K, Travis WD, Centeno JA, Abraham JL, et al. Navy Lung Disease Assessment Program. SAN DIEGO CA: NAVAL HEALTH RESEARCH CENTER; 2004

30. Glazer CS. Occupation, avocation, and interstitial lung disease. Clin Pulm Med. 2011;18(1):20-8. https://doi.org/10.1097/CPM.0b013e318203a42b.

31. Mazzei MA, Sartorelli P, Bagnacci G, Gentili F, Sisinni AG, Fausto A, et al. Occupational lung diseases: underreported diagnosis in radiological practice. Semin Ultrasound CT MR. 2019;40(1):36-50. https://doi.org/10.1053/ j.sult.2018.10.019.

32. Akhter N, Rizvi NA. Interstitial lung diseases misdiagnosed as tuberculosis. Pak J Med Sci. 2018:34(2):338-41. https://doi.org/10.12669/pjms.342.14407.

33. Inai K. Diagnosis of Asbestos exposure-related diseases based on pathological features. Jpn J Lung Cancer. 2009:49(1):83-7. https://doi.org/10.2482/haigan.49.83.

34. Larsen BT, Klein JRH, Hornychova H, Nuti R, Thirumala S, Leslie KO, et al. Pulmonary. Lab Investig. 2013:93(S1):449-71.

35. Preisser AM, Harth V. Allergic bronchopulmonary aspergillosis as an occupational disease - special risk in waste management. Eur Respir J. 2017; 50(suppl 61):PA1226

36. Houle M-C, Holness LD, DeKoven J, Skotnicki S. Additive value of patch testing custom epoxy materials from the workplace at the occupational disease specialty Clinic in Toronto. Dermatitis. 2012;23(5):214-9. https://doi. org/10.1097/DER.0b013e31826f5b70.

37. Kucenic MJ, Belsito DV. Occupational allergic contact dermatitis is more prevalent than irritant contact dermatitis: a 5-year study. J Am Acad Dermatol. 2002;46(5):695-9. https://doi.org/10.1067/mjd.2002.118561.

38. Weber IC, Davis CP, Greeson DM. Phytophotodermatitis: the other "lime" disease. J Emerg Med. 1999;17(2):235-7. https://doi.org/10.1016/S0736-4 679(98)00159-0.

39. Randhawa PS, Mansuri S, Rubin JS. Is dysphonia due to allergic laryngitis being misdiagnosed as laryngopharyngeal reflux? Logoped PhoniatrVocol. 2010;35(1):1-5. https://doi.org/10.3109/14015430903002262. 
40. Rodriguez AV, Kooh M, Guerra LA, Martinez-Lavin M, Pineda C. Musician's cramp: a case report and literature review. J Clin Rheumatol. 2005;11(5):2746. https://doi.org/10.1097/01.rhu.0000182194.80366.49.

41. Ryan WG. Calcific tendinitis of flexor carpi ulnaris: an easy misdiagnosis. Arch Emerg Med. 1993;10(4):321-3. https://doi.org/10.1136/emj.10.4.321.

42. Swathi N, Umadevi J. An undetected intraorbital foreign body after a "trivial"facial injury. J Craniofac Surg. 2014;25(5):1782-3. https://doi.org/10.1 097/SCS.0000000000000905.

43. Mirzaei F, Salehpour F, Shokuhi G, Asvadi Kermani T, Salehi S, Parsay S. An unusual case of intra orbital foreign body; diagnosis, management, and outcome: a case report. BMC Surg. 2019;19(1):76. https://doi.org/10.1186/s12 893-019-0536-2

44. Giannikas KA, Livesley PJ. Penetrating puncture wound due to a grinding machine: a challenge in diagnosis. J Trauma. 1998;44(2):404-5. https://doi. org/10.1097/00005373-199802000-00034.

45. Greenspan A, Gerscovich E, Szabo RM, Matthews J 2nd. Condensing osteitis of the clavicle: a rare but frequently misdiagnosed condition. AJR Am J Roentgenol. 1991;156(5):1011-5. https://doi.org/10.2214/ajr.156.5.2017922.

46. He WJ, Wang DQ, Zhang PP, Fu PL, Li ZJ. The importance of occupational history in clinical thinking from the diagnosis and treatment of a case of Tsutsugamushi disease. Zhonghua lao dong wei sheng zhi ye bing za zhi = Zhonghua laodong weisheng zhiyebing zazhi = Chinese journal of industrial hygiene and occupational diseases. 2019;37(8):639-41.

47. Ross SM. Delayed cognitive and psychiatric symptoms following methyl iodide and manganese poisoning: potential for misdiagnosis. Cortex. 2016; 74:427-39. https://doi.org/10.1016/j.cortex.2015.06.031.

48. Zhang J, Li Z, Wang J, Li H, Si T, Deng L, et al. Misdiagnosis of occupational chronic n-hexane poisoning: an analysis of 16 cases. Zhonghua lao dong wei sheng zhi ye bing za zhi= Zhonghua laodong weisheng zhiyebing zazhi= Chinese journal of industrial hygiene and occupational diseases. 2014;32(12):930-1.

49. Menezes G, D'Souza HS, Venkatesh T. Chronic lead poisoning in an adult battery worker. Occup Med (Oxford, England). 2003;53(7):476-8.

50. Kadu AS, Nampalliwar AR, Pandey AG, Sharma A, Gothecha VK. Lead poisoning: an overlooked diagnosis in clinical practice. Int J Res Ayurveda Pharm. 2012;3(5):639-44. https://doi.org/10.7897/2277-4343.03511.

51. Tezer H, Erkocoglu M, Kara A, Bayrakci B, Duzova A, Teksam O, et al. Household poisoning cases from mercury brought from school. Eur J Pediatr. 2011;170(3):397-400. https://doi.org/10.1007/s00431-010-1317-1.

52. Liu XL, Wang HB, Sun CW, Xiong XS, Chen Z, Li ZS, et al. The clinical analysis of mercury poisoning in 92 cases. Zhonghua nei ke za zhi. 2011;50(8):687-9.

53. Shusterman DJ. Polymer fume fever and other fluorocarbon pyrolysisrelated syndromes. Occup Med. 1993;8(3):519-31.

54. Tonini S, Dellabianca A, Costa C, Lanfranco A, Scafa F, Candura SM. Irritant vocal cord dysfunction and occupational bronchial asthma: differential diagnosis in a health care worker. Int J Occup Med Environ Health. 2009; 22(4):401-6. https://doi.org/10.2478/v10001-009-0038-z.

55. Galdi E, Perfetti L, Pagella F, Bertino G, Ferrari M, Moscato G. Irritant vocal cord dysfunction at first misdiagnosed as reactive airway dysfunction syndrome. Scand J Work Environ Health. 2005;31(3):224-6. https://doi.org/10.5271/sjweh.873.

56. Dupas D, Dagorne MA. Multiple chemical sensitivity: a diagnosis not to be missed. Rev Mal Respir. 2013;30(2):99-104. https://doi.org/10.1016/j.rmr.2012. 06.016 .

57. Chirico F. Adjustment disorder as an occupational disease: Our experience in Italy. Int J Occup Environ Med (The IJOEM). 2016;7(1 January):52-7. https://doi.org/10.15171/ijoem.2016.716.

58. Papakonstantinou D, Riste MJ, Langman G, Moran E. Misdiagnosing Whipple's disease in the young. BMJ Case Rep. 2017;2017:bcr2016218866.

59. Morgan DR. The general practitioners' view. Occup Med (Oxford, England). 1999;49(6):403-5.

60. Dudarev AA, Talykova LV, Odland JO. Occupational diseases in Murmansk oblast: 1980-2010. Int J Circumpolar Health. 2013;72(1):20468. https://doi. org/10.3402/ijch.v72i0.20468

61. Kahan E, Weingarten MA, Appelbaum T. Attitudes of primary care physicians to the management of asthma and their perception of its relationship to patients' work. Isr J Med Sci. 1996;32(9):757-62.

62. Marinides Z, Virgilio GVR. Recurrent facial nerve baroparesis in a military diver: a case report. Undersea Hyperb Med. 2019:46(1):87-90.

63. Fiz Galende J, Dominguez Rodriguez Y, Candel Gonzalez FJ, Cuervo PR. Report of a case of leptospirosis: the importance of asking the patient about workplace factors. Emergencias. 2018;30(1):63-4.
64. Eovaldi B, Dunn DP. Case report: corpus cavernosum thrombosis occurring during a long-range aviation mission initially diagnosed as lymphoma. Mil Med. 2015;180(5):e608-10. https://doi.org/10.7205/MILMED-D-14-00506.

65. Corbett EL, Murray J, Churchyard GJ, Herselman PC, Clayton TC, De Cock $\mathrm{KM}$, et al. Use of miniradiographs to detect silicosis. Comparison of radiological with autopsy findings. Am J Respir Crit Care Med. 1999;160(6): 2012-7. https://doi.org/10.1164/ajrccm.160.6.9903040.

66. Ghio A, Sangani R, Roggli V. Expanding the spectrum of particle-and fiberassociated interstitial lung diseases. Turk Toraks Dergisi. 2014;15(1):1-8. https://doi.org/10.5152/ttd.2014.3950.

67. Taskar VS, Coultas DB. Is idiopathic pulmonary fibrosis an environmental disease? Proc Am Thorac Soc. 2006;3(4):293-8. https://doi.org/10.1513/pats.2 00512-131TK.

68. Laurent F, Paris C, Ferretti GR, Beigelman C, Montaudon M, Latrabe V, et al. Inter-reader agreement in HRCT detection of pleural plaques and asbestosis in participants with previous occupational exposure to asbestos. Occup Environ Med. 2014;71(12):865-70. https://doi.org/10.1136/oemed-2014-1 02336.

69. Wuellner S, Phipps P. Employer knowledge of federal requirements for recording work-related injuries and illnesses: implications for occupational injury surveillance data. Am J Ind Med. 2018;61(5):422-35. https://doi.org/1 0.1002 /ajim.22824

70. Hinchcliffe R. Medical examiner variability. J Laryngol Otol. 1997;111(1):8-14. https://doi.org/10.1017/S0022215100136321.

71. Igata A. Epidemiologic and clinical features of Minamata disease. Environ Res. 1993;63(1):157-69. https://doi.org/10.1006/enrs.1993.1137.

72. Hartman DE. Missed diagnoses and misdiagnoses of environmental toxicant exposure: the psychiatry of toxic exposure and multiple chemical sensitivity. Psychiatr Clin N Am. 1998;21(3):659-70. https://doi.org/10.1016/S0193-953 X(05)70030-7.

73. Black DW. Environmental illness and misdiagnosis-a growing problem. Regul Toxicol Pharmacol. 1993;18(1):23-31. https://doi.org/10.1006/rtph.1 993.1041.

74. Stenton SC, Beach JR, Dennis JH, Keaney NP, Hendrick DJ. Glutaraldehyde, asthma and work--a cautionary tale. Occup Med (Oxford, England). 1994; 44(2):95-8.

75. Ansley L, Kippelen P, Dickinson J, Hull J. Misdiagnosis of exercise-induced bronchoconstriction in professional soccer players. Allergy. 2012;67(3):390-5. https://doi.org/10.1111/j.1398-9995.2011.02762.x.

76. Walusiak J, Palczynski C. Carcinoid behind baker's asthma. Allergy. 2002; 57(10):966-7. https://doi.org/10.1034/j.1398-9995.2002.23832_12.x.

77. Egilman D, Rankin BS. Attorney-directed screenings can be hazardous. Am J Ind Med. 2004;45(3):305-7. https://doi.org/10.1002/ajim.10347.

78. Mayer A, Maier L, Gottschall EB. Mycobacterium AVIUM-Intracellulare Infection and Department Of Labor Criteria For Chronic Beryllium Disease. C52. OCCUPATIONAL LUNG DISEASE. American Thoracic Society International Conference Abstracts: American Thoracic Society; 2011. A4779A4779.

79. Kales SN, Lee EC. Pseudo-latex allergy associated with "latex" paint exposure: a potential cause of iatrogenic disability. J Occup Environ Med. 2006;48(1): 83-8. https://doi.org/10.1097/01.jom.0000184882.67947.58.

80. Bruze M, Hamada H, Dahlin J, Duner K, Persson L. A positive cobalt spot test falsely indicating an occupational allergic contact dermatitis caused by cobalt. Contact Dermatitis. 2013;69(3):172-5. https://doi.org/10.1111/cod.12 058.

81. Bouzgarrou L, Omrane A, Chebbeh W, Boussarssar I, Kellala I, Belkhiria K, et al. Diagnosis mistaken: a non-negligible reason for rejecting the declaration of occupational dermatitis. World Allergy Organization J. 2020; 13(8):100220.

82. Poole CJ. Cold haemagglutinin disease misdiagnosed as hand-arm vibration syndrome. Occup Med (Oxford, England). 2008;58(3):219-21.

83. Chan JC, Tiong WH, Hennessy MJ, Kelly JL. A Guyon's canal ganglion presenting as occupational overuse syndrome: a case report. J Brachial Plex Peripher Nerve Inj. 2008;3(1):4.

84. Waljee JF, Ring D. Diagnosis and treatment of carpal tunnel syndrome in low-prevalence circumstances. J American Acad Orthop Surg. 2018;26(16): 573-5. https://doi.org/10.5435/JAAOS-D-17-00575.

85. Griffin BR, Butler-Dawson J, Dally M, Krisher L, Cruz A, Weitzenkamp D, et al. Unadjusted point of care creatinine results overestimate acute kidney injury incidence during field testing in Guatemala. PLoS One. 2018;13(9):e0204614. https://doi.org/10.1371/journal.pone.0204614. 
86. Behrman A, Schmid DS, Crivaro A, Watson B. A cluster of primary varicella cases among healthcare workers with false-positive varicella zoster virus titers. Infect Control Hosp Epidemiol. 2003;24(3):202-6. https://doi.org/10.1 086/502187.

87. Schaumburg HH, Albers JW. Pseudoneurotoxic disease. Neurology. 2005; 65(1):22-6. https://doi.org/10.1212/01.WNL.0000169015.79751.F4.

88. Greve KW, Bianchini K, Black FW, Heinly MT, Love JM, Swift DA, et al. The prevalence of cognitive malingering in persons reporting exposure to occupational and environmental substances. Neurotoxicology. 2006;27(6): 940-50. https://doi.org/10.1016/j.neuro.2006.06.009.

89. Greenland S, Robins JM. Epidemiology, justice, and the probability of causation. Jurimetrics. 1999:40:321.

90. Graber ML, Franklin N, Gordon R. Diagnostic error in internal medicine. Arch Intern Med. 2005;165(13):1493-9. https://doi.org/10.1001/archinte.165.13.1493.

91. Croskerry P. A universal model of diagnostic reasoning. Acad Med. 2009; 84(8):1022-8. https://doi.org/10.1097/ACM.0b013e3181ace703.

92. Rosenstock L, Rest KM, Benson JA Jr, Cannella JM, Cohen J, Cullen MR, et al. Occupational and environmental medicine: meeting the growing need for clinical services. N Engl J Med. 1991;325(13):924-7. https://doi.org/10.1056/ NEJM199109263251305.

93. LaDou J, Harrison R. Current occupational \& environmental medicine. New York: McGraw-hill; 2007.

94. Kolk A, Pinkse J. Stakeholder mismanagement and corporate social responsibility crises. Eur Manag J. 2006;24(1):59-72. https://doi.org/10.1016/j. emj.2005.12.008

95. Norvell D. Study types and bias-Don't judge a study by the abstract's conclusion alone. Evid Based Spine Care J. 2010;1(02):7-10. https://doi.org/1 0.1055/s-0028-1100908.

\section{Publisher's Note}

Springer Nature remains neutral with regard to jurisdictional claims in published maps and institutional affiliations.

Ready to submit your research? Choose BMC and benefit from:

- fast, convenient online submission

- thorough peer review by experienced researchers in your field

- rapid publication on acceptance

- support for research data, including large and complex data types

- gold Open Access which fosters wider collaboration and increased citations

- maximum visibility for your research: over $100 \mathrm{M}$ website views per year

At $\mathrm{BMC}$, research is always in progress.

Learn more biomedcentral.com/submissions 